\title{
LOS PROYECTOS EDUCATIVOS INSTITUCIONALES Y LA FORMACIÓN DE DOCENTES ${ }^{1}$
}

\section{Gloria Calvo $^{2}$}

\begin{abstract}
Este texto quiere señalar algunas de las posibilidades derivadas de los Proyectos Educativos Institucionales, entendidos como una nueva forma de gestión pedagógica que parte de reconocer y que busca "responder a situaciones y necesidades de los educandos, de la comunidad local, de la región y del país" (decreto 1860 de 1994). Igualmente muestra en qué medida, la gestión centrada en los PEI permite repensar los procesos pedagógicos y deriva en retos para la formación docente al exigir a un maestro que tome decisiones, que participe - con voz y acción-y que sea capaz de buscar y trabajar con una comunidad educativa ampliada.
\end{abstract}

La educación en Colombia no es ajena a los problemas relacionados con la calidad y la equidad del sistema educacional, menos aún cuando los índices de cobertura para los cinco años iniciales de la enseñanza básica alcanzan niveles cercanos al $80 \%$. Sin embargo, las evaluaciones en español y matemáticas repodadas por el Sistema Nacional de Evaluación y recogidas en Saber (1991), además de algunas más recientes efectuadas por el Instituto Colombiano para el Fomento de la Educación Superior-ICFES (1994) a través de su División de Pruebas, ponen de manifiesto diferencias significativas respecto de regiones, contextos culturales y tipo de establecimientos, entre otros. Podría afirmarse que en Colombia la calidad de la educación está asociada con múltiples factores que hacen cada vez más difícil equiparar años de escolaridad y conocimientos.

\section{El contexto normativo de los PEI en Colombia}

Sensible a las deficiencias en materia de calidad y equidad señaladas anteriormente y recogiendo la voluntad del constituyente, expresada en los artículos 73 y 77 de la Constitución del 91, la Ley General de Educación - ley 115 de 1994- concreta la autonomía escolar a la que aludían los citados artículos de la Carta Magna en los Proyectos Educativos Institucionales - PEI. Estos son concebidos como una estrategia para mejorar la calidad de la educación y lograr la formación integral del educando. Por su parte, el decreto 1860 de 1994, al reglamentar la ley anterior define que:

"Todo establecimiento educativo debe elaborar y poner en práctica, con la participación de la comunidad educativa, un Proyecto Educativo Institucional que exprese la forma como se ha decidido alcanzar los fines de la educación, definidos por la Ley, teniendo en cuenta las condiciones sociales, económicas y culturales de su medio" (subrayados nuestros).

En concordancia con este nuevo escenario, la política social sectorial plasmada por el Departamento Nacional de Planeación y la Presidencia de la República en el documento titulado "El Salto Educativo" (1994), generalizó esta estrategia para todas las instituciones del país como la posibilidad de afectar las deficiencias relacionadas con la calidad y equidad de la educación.

\footnotetext{
1 Ponencia presentada en el Seminario "Nuevas formas de enseñar y de aprender", organizado por OREALC-UNESCO. Santiago, noviembre de 1995.

${ }^{2}$ Docente-Investigadora de la Universidad Pedagógica Nacional. 
¿Es este contexto legal el resultado de una reflexión propia del país sobre una segmentación cada vez más palpable de un sistema educacional? ¿o es, como lo señalan algunos investigadores (Alvarez, 1995) una respuesta a los criterios y señalamientos de las agencias (CEPAL - UNESCO, 1992: 131) que ven en la concepción de la escuela como un proyecto - intelectual e institucional - la manera de garantizar la identidad de la misma y, en consecuencia, una forma de incidir en la calidad de los aprendizajes?

Esta polémica ha estado presente en las reflexiones plasmadas en algunas revistas educativas que circulan en el país (Pretextos Pedagógicos, No. 2; Educación y Cultura, No. 38), pero, sin lugar a dudas, esta polémica no ha afectado la dinámica de la elaboración de los PEI. Por una parte, debido a la existencia de procesos de innovación presentes en el país desde hace casi una década y por otra, dados los plazos perentorios y la amenaza de sanción estipuladas por el mismo decreto 1860, que anunciaba la cancelación de las aprobaciones a las instituciones que no hayan reflexionado sobre sus fines y propósitos, esto es, que no tengan consignada su visión y su misión en abril de 1995 y el Proyecto Educativo en su totalidad en el primer trimestre de 1997.

\section{La práctica de los PEI en Colombia}

Frente a la elaboración de los Proyectos Educativos Institucionales - PEI - los maestros colombianos se vieron enfrentados a un reto que por lo menos suscitó dos actitudes diferentes, evidenciables en testimonios: una primera, asumida por aquellos docentes familiarizados con procesos de innovación, y sobre todo con el manejo de Proyectos: "El trabajo por proyectos es también una estrategia pedagógica que nos permite avanzar, reconociendo los procesos que se van generando por nuestra acción es, esencialmente, una intención compartida para transformar la realidad para incidir sobre situaciones concretas" (Martínez, 1995: 16); y una segunda, de asombro que pone al desnudo las limitaciones y que reconoce las nuevas exigencias: "la elaboración del PEI produce un sentimiento contradictorio entre los docentes. Por una parte, la responsabilidad y la complejidad de las decisiones y, por otra, la posibilidad de decidir sobre el propio trabajo, tanto en el ámbito organizativo como en el de los contenidos de la enseñanza, ya que deben ser discutidos a nivel colectivo" (Cubides, 1995: 26). "Los PEI tomaron a los docentes por sorpresa, sin la debida motivación y capacitación para afrontarla, creando, por lo general, confusión, desánimo o rechazo" (Escuela Filodehambre, 1995: 31).

Como respuesta a estas actitudes, rápidamente el Ministerio ofreció apoyo y, junto con la asesoría ministerial, las ofertas de expertos, seminarios, textos y manuales, producidos por autores individuales, colegios privados y editoriales han inundado el mercado (Lucio, 1995:21). Incluso ya es posible identificar una metodología fácilmente establecida que desdibuja los procesos de participación y de consenso implícitos en la definición y formulación de los Proyectos Educativos Institucionales. Esta "cultura del facilísimo", sin lugar a dudas, va a generar una dinámica diferente de la esperada en cuanto que no compromete a la comunidad educativa en un proceso encaminado a mejorar la gestión pedagógica.

En aquellas instituciones familiarizadas con el trabajo por proyectos y con alguna trayectoria en el manejo de innovaciones pedagógicas, el PEI ha otorgado carta de ciudadanía a sus esfuerzos. En este sentido es significativo encontrar testimonios que afirman: "nos adelantamos a la Reforma", tal como lo han expresado los maestros de un colegio técnico de un municipio de la región cafetera del país y que quiere manifestar con esta afirmación que el proyecto curricular implementado en esa institución recogía, desde antes de la ley, el sentido y las necesidades de la comunidad educativa. Más aún, los 
testimonios docentes hablan de a consensos y compromisos con autoridades del orden municipal y departamental con el objeto de hacer viable la propuesta garantizando, al menos, la planta docente necesaria para el proyecto (Alegría de Enseñar, No. 22: 63). Casi que podría afirmarse que aquellos procesos de elaboración de PEls más avanzados, se han anclado en dinámicas innovadoras generadas con anterioridad.

\section{Retos para la cualificación de los PEI}

Un somero inventario recogido en las revistas de mayor circulación entre el magisterio permiten verificar esta hipótesis: el número 38 de la Revista de la Federación Colombiana de Educadores - FECODE -recoge las experiencias en la elaboración del PEI en la Escuela Filodehambre, en el departamento del Huila, institución con una amplia trayectoria en manejo de relaciones con la comunidad a través de la Experiencia en la Escuela Popular Claretiana y galardonada con uno de los premios a las innovaciones educativas que otorga la Secretaria del Convenio Andrés Bello - SECAB (Educación y Cultura, No. 38:31-36). Por su parte, la edición No. 22 de Alegría de Enseñar, al hacer una reseña sobre lo que pasa con el PEl en el país, da mayor importancia al trabajo realizado desde hace cinco años por la Escuela Nueva Delhi, institución situada al sur oriente de la ciudad de Bogotá. Allí, los docentes han plasmado una propuesta curricular alrededor de tres ejes: el lenguaje, la estética y los derechos humanos. Con esta propuesta han buscado responder al contexto de los alumnos y hacer que ellos logren aprendizajes significativos, que recuperen el placer por la creación estética y el respeto de sí mismos, en la diferencia (Alegría de Enseñar, No. 22: 22-30). Otras experiencias significativas en la elaboración del PEI en 10 escuelas de la capital estuvieron aglutinadas alrededor de un Proyecto de Investigación dentro de la Problemática de la Educación en Derechos Humanos, que estuvo liderada por un destacado grupo de intelectuales. La idea, en el momento, es generalizar los logros de las escuelas a partir de una propuesta de material producido colectivamente.

Sin embargo, aun para aquellos procesos más avanzados, se impone enfatizar en el hecho de que los Proyectos Educativos Institucionales son una construcción colectiva (MEN, 1994) que debe comprometer la intencionalidad de cada uno de los miembros de la comunidad educativa: maestros, alumnos, padres, la localidad en la cual se encuentra la escuela, y más aún: que es deseable que el PEI participe de los planes de desarrollo locales, municipales y regionales. Sólo en este sentido el PEI podrá "dibujar el mapa por el que la comunidad educativa camina hacia el futuro" (Peña, L. B., 1995: 8). De otra suerte seguiríamos trabajando alrededor de proyectos pedagógicos de aula.

La comunidad educativa también necesita tener presente que el PEI comienza con la escritura del documento, producto de un diálogo y una negociación. Su formulación debe responder a los postulados de una pragmática comunicativa: sinceridad, rectitud, verdad. En tal sentido los PEI pueden ser conocidos, cuestionados, verificados. En ellos toda acción tiene un sentido (Bustamante, 1995a; 1995b).

Pensar que el PEl termina en el momento en que se entrega el documento en la Secretaria o en el Ministerio o cuando quede registrado en el Sistema Nacional de Información en 1997, es pensar la estrategia de los Proyectos Educativos Institucionales bajo una óptica instrumental que poco o nada aporta a la calidad y la equidad del sistema educacional, Olvidaría igualmente que en los PEI se enfatiza la acción sobre los sucesos sociales o sobre los sistemas valorales (Bustamante, 1995b). 
Una institución escolar que actuara sólo bajo la presión del cumplimiento de la norma, no habría superado el nivel heterónomo de la gestión, y en tal sentido no habría hecho uso del PEI como posibilidad de reivindicación de la autonomía de los procesos pedagógicos a partir del sentir de los actores de la comunidad educativa: "Una verdadera descentralización significa entonces autonomía, sentido de proyecto, identidad institucional e iniciativa y capacidad de gestión radicada dentro de los propios centros educacionales" (CEPAL-UNESCO, 1992: 131).

Pero, más allá de los discursos de las agencias, el PEl puso a reflexionar a los docentes sobre qué implica aprender y qué significa enseñar. Así mismo señaló que hay otras formas, diferentes de las institucionalizadas para aprender y que el afecto, dentro del aprendizaje, es importante. Igualmente ha mostrado que para pensar la escuela se necesita tiempo y que una sola jornada no es suficiente. También ha permitido evidenciar que hay diferentes niveles en la elaboración conceptual sobre los PEl y que su concepción y desarrollo van más allá de los tiempos estipulados por las normas, a la vez que exigen procesos de sistematización y de investigación.

Para aquellos docentes más comprometidos el formato exigido para consignar los PEI es tedioso; lleva implícito el equiparar datos con procesos de investigación y supone conocimientos que el PEI mismo tiene como finalidad construir. Por estas razones los docentes identifican los formatos y el tiempo como los dos mayores obstáculos para la elaboración del PEl. Algunos maestros, con más trayectoria en la formulación de Proyectos, señalan que el logro del consenso, el trabajo con todos los sectores de la comunidad educativa, el reconocimiento de los diferentes niveles de conceptualización frente a un problema, amén de la participación de padres y alumnos, son las dificultades de mayor trascendencia para la formulación de los PEI y para lograr aquello solicitado hasta la fecha, a saber, la visión y la misión de la institución, además de los acuerdos para la convivencia y el gobierno escolar.

\section{Los Proyectos Educativos Institucionales: campo dinámico y en gestación}

Dentro del Programa para promoción de la Reforma Educativa -PREAL— coordinado en Colombia por el Instituto Ser de Investigación, se efectuó en octubre de 1995 una convocatoria nacional con el fin de identificar la articulación del PEl y el currículo. El MEN, la Fundación Presencia y el mismo SER, seleccionaron 7 proyectos entre más de 30 presentados.

Un análisis de tales proyectos desde la perspectiva de las relaciones entre procesos de innovación y formulación de PEls permite mantener la hipótesis de que los mayores avances en dicha materia están fundamentados en procesos previos de innovación.

EI PEI del Colegio Comunitario Luis Carlos Valencia, en Villapaz -Quinamanyó, en el municipio de Jamundí en el Valle del Cauca, se basa en un trabajo previo de investigación-acción titulado "Curriculum y comunidad: una experiencia de innovación educativa". En él contaron con la asesoría de Ceneyra Chávez y Maria Cristina Navarrete, quienes orientaron un proceso de construcción curricular con los docentes para su mismo proceso de formación. ${ }^{3}$

El Colegio Comentario Luis Carlos Valencia, ha venido acomodando su estructura hasta llegar a un órgano máximo de dirección: la asamblea de profesores. De ella

\footnotetext{
${ }^{3}$ Una reseña de este proyecto está consignada en el RAE, especializado en Formación Docente, producido por REDUC regional para la UNESCO.

Digitalizado por RED ACADEMICA
} 
dependen, como asesores, el representante legal (rector) y el secretario del colegio. Existe un coordinador para disciplina y bienestar, otro para proyectos con la comunidad, un coordinador de talleres y otro agropecuario. Estos coordinadores conforman un comité del cual dependen las acciones de profesores, estudiantes y la comunidad educativa en general.

Para abordar el Plan de Estudio se tuvo como eje el establecimiento de las relaciones entre intencionalidad educativa y los aspectos culturales de los involucrados en el proyecto.

Queda el temor de que algunos proyectos pedagógicos se estén asimilando a los Proyectos Educativos Institucionales, dejando de lado el proceso de negociación de saberes que implica la construcción participativa de un proyecto institucional. No obstante, algunos PEl empiezan a trabajar en esta dinámica. El del Colegio Marco Fidel Suárez de Barranquilla, ha desarrollado acciones sistemáticas con la comunidad e inclusive ha diseñado materiales para procesos de sensibilización encaminados a la conceptualización sobre el sentido de la escuela y su relación con la comunidad.

En el Proyecto Educativo Campoamor, apoyado por la Fundación Social y que data de 1897, la construcción del PEI ha sido participativa: se ha consultado y tenido en cuenta las ideas presentadas por miembros de los diferentes estamentos de la comunidad. Incluso, los padres de familia dirigen un subproyecto de Bienestar Infantil y están organizando un círculo de estudio para potenciar su participación en el gobierno escolar.

Como elemento interesante en esta escuela se disemina el modelo de PEI, el cual se combina con proyectos pedagógicos en áreas específicas (área socio-afectiva y valoral; comprensión y expresión de la lengua oral y escrita; desarrollo de la creatividad y animación cultural). Sin embargo, los retos en cuanto a contenidos mínimos, articulación curricular entre niveles - el valor, por ejemplo, de los conocimientos universales para una propuesta curricular - no aparece. Tampoco la pertinencia del currículo en el contexto local, sus relaciones con la vida cotidiana y su articulación con los saberes necesarios para la vida dentro de las sociedades modernas.

\section{Posibilidades que ofrecen los PEI en Colombia}

Los proyectos educativos institucionales se mueven entre la dicotomía del mandato legal y la posibilidad de ser una herramienta que legitime algunos de los derechos consagrados en la Constitución del 91, en especial los relacionados con la participación, la democracia, la autonomía y la autogestión (Bustamante, 1995a: 38). Ellos podrían conformar campos de fuerzas donde confluyan las tendencias educativas mundiales y las particularidades de la cultura nacional (Alvarez, 1995:28). En este sentido la educación tendría la posibilidad de convertirse en un Proyecto Cultural que recoja las expectativas de la comunidad y las resignfique dentro de contextos ampliados, es decir, parta de lo local y lo confronte con códigos donde prime lo interdisciplinario, lo transversal. Por esto, el mismo autor no vacila en afirmar que es en el terreno de los PEI donde se va a definir, sin lugar a dudas, lo que ha de ser la educación en los próximos años (Alvarez, 1995: 34). Así entendidos los Proyectos Educativos Institucionales, permiten que lo educativo se relacione con otras formaciones culturales, históricamente determinadas, y que el "oficio de enseñar" se articule con un proyecto sociocultural general en momentos y contextos específicos. En este sentido se garantizaría la autonomía del individuo, entendida como la posibilidad de construir relaciones críticas en diferentes contextos socioculturales (De Tezanos, 1992: 157 y 158). 
Por otra parte, los PEI van a permitir, como de hecho ha venido ocurriendo, el impulso de innovaciones individuales o colectivas de maestros, cambio en las prácticas educativas y pedagógicas, posibilidades de cambios en unas comunidades, en unas prácticas ... en unos maestros (Noguera, 1995: 19-26). En el ejercicio de la autonomía escolar que posibilitan los PEI pueden llegar a constituirse, en algunas instituciones concretas, mecanismos de experimentación de nuevas posibilidades para las prácticas educativas, la descentralización de la enseñanza y el aprendizaje, el desestímulo al autoritarismo del maestro, en fin, la apertura de la escuela a la comunidad.

Estas consideraciones plantean la conveniencia de ver en la formulación del PEI una estrategia deformación y más allá del formato que requiere y a veces exige respuestas inmediatas, asumir la elaboración del PEI como un proceso de investigación, sobre lo pedagógico, que permita identificar logros y vacíos más que respuestas certeras e inmediatas. "Lo importante de los PEl es que la escuela investigue sobre sí misma y no que llene una serie de datos sobre recursos económicos, planta física, repitencia, puntaje en los exámenes de Estado" (Bustamante, 1995b: 8), datos que si bien son importantes muchas veces llevan a que lo innovativo sea asimilado a cambios físicos en la institución (Moreno, 1994), como lo demuestra un análisis de las solicitudes de legalización de procesos de innovación ante la oficina del ramo en el Ministerio de Educación Nacional.

El reto para los PEl en el país, reto del que también participan el Ministerio, las Secretarias de Educación, las universidades y los distintos organismos encargados de asesorar, evaluar y vigilar la elaboración de los proyectos y su puesta en marcha, es "no permitir que los excesivos formalismos legales o la tramitología administrativa, tan persistentemente arraigados en nuestra cultura, vayan a imperar sobre el verdadero sentido de los PEIs" (Peña, 1995: 9). Así mismo se hace necesario velar porque el sentido colectivo y participativo de los PEI no sea asimilado a un proceso tradicional de planeación (Bustamante, 1995), tendencia que parece estar manifestándose en algunas instituciones educativas.

\section{Los PEI como estrategia de participación}

El PEl es un factor fundamental dentro de la relación escuela-comunidad. En consecuencia, expresión de la autogestión y la autoconstrucción. De allí que los criterios para su evaluación dependan del esfuerzo y del trabajo de cada comunidad, ya que cada una es distinta y en cada una esfuerzo y trabajo responden a sentidos diferentes. "Lo que una comunidad se plantea como interrogante puede haber sido resuelto por otra anteriormente" (Bustamante, 1 995b: 10).

Por otra parte, la estrategia participativa derivada de los Proyectos Educativos Institucionales tiende a romper el carácter solitario del trabajo docente (PIIE, 1994:57) y adiciona espacios para pensar, para discutir, para dudar, para crear en equipo y para que las responsabilidades educativas no sean sólo del maestro.

EI PEI exige un proceso de sistematización, socialización y evaluación continua que permita reconstruir su camino, signado de incertidumbre. Las comunidades educativas podrían diseñar estrategias de interevaluación en donde las instituciones pudieran contrastar sus logros y dificultades con los de otras similares. Esta participación de la comunidad en lo que hasta ahora ha sido propio de la escuela llevaría a mayores exigencias en cuanto a la calidad, equidad y eficiencia del sistema educativo y buscaría, como es el espíritu de la Ley General de Educación, conferir autonomía a las entidades territoriales y a las instituciones educativas de tal forma que la educación esté acorde con 
la realidad local y que a través del trabajo colectivo se trascienda la rigidez de la escala jerárquica, construyendo y reconstruyendo conocimientos con otros. En esta "escuela participativa" (Rodríguez, 1990: 31) todos influyen en su funcionamiento; nadie se siente al margen de lo que sucede; cada uno aporta su propia individualidad.

\section{Los PEI como nuevas formas de aprender}

Como consecuencia de la autonomía pedagógica facilitada y propiciada por los Proyectos Educativos Institucionales, las escuelas pueden establecer Planes de Estudio, entendidos como una guía general de tiempos y una secuencia para acceder al conocimiento. La descentralización pedagógica está encaminada a que la institución educativa asuma las implicaciones derivadas de las preguntas sobre qué aprender; por qué, para qué, cómo. "El énfasis ya no está en la enseñanza sino en la adquisición de las herramientas básicas para aprender a aprender" (Coy, 1995: 13-18).

En tal sentido lo prioritario frente al aprendizaje es la construcción de conocimiento, la producción y la negociación de sentido. Aprender, en este nuevo contexto significa la apropiación y la construcción de universos de sentido (Salazar, 1995: 40). Casi que podría vislumbrarse en este contexto una escuela para el pensamientos donde se enseñe prioritariamente a los niños a expresarse, a descubrir, a solucionar problemas, a dar su opinión (Rodríguez, 1990:20) y en donde la creatividad, la laboriosidad, la autonomía y el progreso nos recordarían los principios de la pedagogía activa y la Escuela Laboriosa de Dewey.

Los maestros de la Escuela Filodehambre (1995: 36) expresaban bastante bien este cambio al mostrar que el PEI los obliga a revisar continuamente sus conocimientos, los contenidos curriculares, las metodologías y los procesos pedagógicos. Esta provisionalidad ha puesto a pensar a los maestros, quienes reconocen que con el PEI no hay currículos establecidos y que el trabajo por proyectos los lleva a continuar cambios y a romper con la concepción de aprendizaje como un proceso repetitivo y memorístico. Estas nuevas formas de aprender estarían más próximas a las demandas que se le hacen a la educación en nuestro tiempo (Abrile de Vollmer, 1994: 37) y reconocerían el papel de la subjetividad en los procesos de apropiación y construcción de conocimiento. Reconocerían, por lo demás, el interjuego que significa el aprender: en los procesos de instrucción y aprendizaje se articulan el conocimiento, el contexto y las subjetividades de los actores involucrados (Remedi, 1992: 25).

Las modificaciones que introduce el PEI pasan también por los cambios que favorecen en la cultura escolar, entendida como las formas en que son significadas, al interior de la escuela, las relaciones entre los actores de la realidad educativa; las relaciones de estos actores con el conocimiento; la forma y contenido de dichos actores como tales; las formas y estrategias de la relación de la unidad educativa y sus integrantes con las demás esferas del mundo de la educación (PIIE, 1994:24). El Proyecto Educativo Institucional pasa tanto por las necesidades e intereses de los niños como por los pensamientos y visiones de los distintos estamentos de la comunidad educativa, pero, sobre todo, por las prácticas docentes y por la forma como éstas articulan y vivencian cualquier proceso de innovación y cambio.

¿Cómo responder, desde la formación inicial de docentes, a las exigencias derivadas de los procesos de descentralización pedagógica? La experiencia recogida en la puesta en marcha de los Proyectos Educativos Institucionales en Colombia permite algunos comentarios. 
A partir de la Ley General de Educación, los maestros de Colombia deben ser formados para el ejercicio de la autonomía intelectual en una cultura pedagógica, universal y nacional (MEN, 1995:12). Según sus propuestas, lo pedagógico y lo didáctico se articulan para que al docente se le abra un camino que dinamice su actuar en múltiples campos: el cotidiano, el experimental, el conceptual y el comunitario. Igualmente insiste para que el maestro, más que un funcionario, sea un analista de los procesos de formación, aprendizaje, educación y enseñanza. Reconoce, igualmente, la articulación de la pedagogía con una praxis social específica cual es la enseñanza, sus condiciones de existencia, sus reglas y sus transformaciones históricas (Tezanos, 1992: 157).

Este reconocimiento de la Pedagogía como un campo del saber y del maestro como actor y creador de la enseñanza lleva al reconocimiento del docente en su trabajo, "en donde el ritmo, la referencia, la reconstrucción no la define el referente pautado por la institución, sino la dinámica, el hacer de la interacción que se establece por los sujetos que participan en la clase" (Remedi, 1992:27).

De las anteriores consideraciones se deriva que para implementar políticas educativas que incidan efectivamente en el mejoramiento de la calidad de la educación es imprescindible tener presentes ciertos rasgos de la cultura escolar que pasan por las prácticas docentes y que están relacionadas con los modelos de enseñar y de aprender en los que fue formado el maestro.

Por ejemplo, ¿Cómo hacer que el diagnóstico de las necesidades de la escuela, su visión y su misión no sean las de una comunidad idealizada o determinada por aquello que cree el docente como pertinente para la misma? ¿Cómo hacer que lo administrativo se integre a los procesos pedagógicos? ¿Cómo, finalmente, hacer que el aprendizaje se logre en la escuela?

Un estudio reciente, asociado a la implementación de la descentralización en Chile y realizado por el Programa Interdisciplinano de Investigaciones en Educación (PIIE, 1994), señala algunos rasgos de la cultura escolar que pasan por el docente y sobre los cuales fue posible reflexionar a partir de los talleres implementados por los investigadores dentro de dicho proceso investigativo.

El estudio del PIIE llama la atención sobre estas conclusiones: el docente trabaja aislado: no confronta sus prácticas, logros o dificultades con sus colegas: no reconoce su saber, al primar en él una concepción que equipara conocimiento y autoridad; tampoco acepta con facilidad que el conocimiento es un proceso subjetivo, que implica construcción y que no siempre es traducible en formas verificables. Finalmente, que ante cualquier estrategia de cambio, el docente trata de asimilar las nuevas propuestas a aquellos conocimientos o prácticas previas, como silo nuevo le generara un "vacío de saber" que le restara seguridad en su profesión.

Con facilidad pudiera afirmarse que esta forma de ser y actuar corresponde al papel funcional que se le ha asignado social e institucionalmente al docente. La preocupación por los métodos y por las técnicas, por la evaluación estandarizada de los aprendizajes más que por los ritmos y modalidades personales, la preocupación por los contenidos homogéneos más que por las especificidades culturales, han hecho que el maestro no potencie su práctica y que desplace al mundo administrativo elementos relacionados con los procesos de enseñanza, que hacen más significativos los aprendizajes. 
Cuando el maestro comprende que el alumno es un sujeto con procesos psíquicos autónomos, no fáciles de observar, que puede actuar sobre su entorno y que a la vez puede ser transformado en su interioridad, el docente es capaz de reconocerse a sí mismo y percibir su quehacer como agente que puede producir profundos cambios en el sujeto y le surge la necesidad de comprender el fenómeno del aprendizaje (Cerda, 1994).

Pero esta nueva forma de ver y comprender la práctica implica una vigilancia crítica sobre su quehacer (Cárdenas, 1994:14), donde es necesario reconocer y elaborar ese contenido biográfico con el fin de que sea claro para el maestro que en los procesos pedagógicos lo subjetivo se pone en juego (Remedi, 1992: 25) y que con su práctica el docente bloquea, facilita, orienta y/o promueve los aprendizajes de los otros, es decir, que los sujetos median en la relación con el conocimiento (Edwards, 1995: 14).

Sin embargo, es necesario reconocer que la estrategia misma del PEI implica un proceso continuo de formación de la comunidad educativa, en general, y del maestro, en particular. Igualmente posibilita el desarrollo de la práctica docente desde núcleos problémicos presentes en la escuela, en ejercicios didácticos, en las innovaciones o en las mismas negociaciones con la comunidad. En este sentido la estrategia de formar docentes articulados a los procesos derivados dc la formulación, puesta en marcha y seguimiento de los proyectos educativos institucionales propende, tal como lo exigen los nuevos lineamientos sobre formación docente, al logro de "una solidez en lo pedagógico, lo disciplinario, lo axiológico y lo ciudadano" (MEN, 1995:26).

Por otra parte, y siguiendo este mismo documento, las relaciones de la escuela con la comunidad exigen una "territorialización del maestro" (MEN, 1995: 23), de tal manera que reconozca las prácticas y los saberes existentes en una determinada región, como códigos configurados por el lenguaje y la cultura en el mundo de la vida, que le darán pertinencia y sentido de identidad con la comunidad y con el territorio que habita. Así, la enseñanza podría entenderse como una acción comunicativa, transcultural, trasdisciplinar y crítica (MEN, 1995: 23).

Más específicamente, la formación de docentes dentro de los procesos derivados de la descentralización pedagógica y puesto de presente por la dinámica generada por los Proyectos Educativos Institucionales, comprendería estos aspectos:

Formación para la interpretación biográfica: mirarse como sujeto y reconocerse como educador. "Reflexión en y sobre la acción", proponían los maestros de la Escuela Filodehambre (1995: 36).

- Formación para la función pedagógica: interpretar el mundo escolar y problematizarlo.

- Formación de competencias morales y sociales tendiente a una Escuela de los Valores (Rodríguez, 1995: 53): construcción de mínimos éticos. Pueden incluirse talleres de reflexión valoral, técnicas para el manejo del debate, resolución de conflictos.

- Formación para construir relaciones alternativas: desconcentrar el poder; motivar la participación ciudadana.

- Formación para la gestación de los PEI, su seguimiento, sistematización y evaluación, que incluya manejo de técnicas para la sistematización de experiencias e innovaciones, ya que el PEI exige un proceso de paulatinos logros y acuerdos. Sólo mediante el conocimiento de su proceso será posible tomar decisiones que incidan en el mejoramiento de la calidad de la educación. 
- Formación organizacional que incluya conocimiento de las instituciones, dinámica de éstas, evolución a partir del PEI, marcos legales de la educación en el país.

- Formación en el manejo de proyectos: formulación —evaluación —negociación — costos.

- Formación para el manejo de los procesos de aprendizaje: tiempos, espacios, actividades, metodologías, así como para evaluarlos.

- Formación para la evaluación de procesos y para el manejo de la evaluación cualitativa: para la coevaluación y heteroevaluación: el problema de evaluar en los niños su apropiación de conocimientos está aún sin resolverse por que, en la medida en que reflexionamos seriamente sobre el tema, nos convencemos más de la relatividad de nuestras apreciaciones" (Escuela Filodehambre, 1995: 35).

- Formación para escribir y comunicar que deriven en la capacidad de recuperar saberes ya internalizados y poder explorar en fuentes diversas para analizarlos y actualizarlos en dinámicas interpretativas específicas. Así el maestro entrará a construir y a participar en redes intertextuales e intercognitivas (Niño, 1995: 180).

A manera de síntesis, podría enunciar algunos postulados sobre lo que ha sido la experiencia de la puesta en marcha de una descentralización, enfocada desde lo pedagógico en Colombia:

- Los Proyectos Educativos Institucionales parecen fijar los campos de fuerza donde se contextualizan los discursos de las agencias y las demandas locales a la educación.

- Más allá de los discursos-oficiales o internacionales-, los PEI ofrecen la oportunidad para la participación y para que la escuela rompa el muro que por mucho tiempo la ha mantenido alejada de la comunidad.

- Los Proyectos Educativos Institucionales van a poner de manifiesto el desarrollo desigual de las instituciones educativas. Los PEI más avanzados hasta el momento están anclados en procesos de innovación y en la trayectoria que tienen algunos docentes en la formulación y trabajo por Proyectos.

- Es de preverse que en las instituciones más renuentes al cambio el proceso de elaboración de los PEI va a tomarse como un proceso rutinario y formal, fácilmente asimilable a los rituales tradicionales de la planeación educativa.

Alrededor de ciertos PEI pueden expresarse algunos rasgos de la cultura nacional, si éstos son asumidos como un proceso que no tenga en cuenta las negociaciones de sentido que deben llevarse a cabo entre los diferentes actores de la comunidad educativa. La "cultura del facilismo" puede verse alimentada por la producción acelerada de manuales para la elaboración del PEI y por modelos que olviden la especificidad de los contextos regionales y culturales.

- Los PEl generarán, sin lugar a dudas, procesos paulatinos de cambio en las prácticas educativas y pedagógicas. Exigirán, en el mediano plazo, un amplio debate alrededor de los contenidos de los planes de estudio y un acuerdo sobre los criterios de logro y eficiencia. 
- Se hace necesario hacer hincapié en el sentido participativo de los PEI para que éstos se diferencien de los Proyectos Pedagógicos de aula y para que la escuela recupere su papel protagónico dentro de la comunidad.

\section{Bibliografía}

Abrile de Vollmer, Maria Inés. "Nuevas demandas a la educación y a la institución escolar y la profesionalización de los docentes", en Revista Iberoamericana de Educación, Madrid, OEl, mayo-agosto. No. 5, 1994.

Alvarez, Alejandro. "Proyecto Educativo Institucional, respuesta de la escuela a una nueva época", en Proyectos Pedagógicos, No. 2, Bogotá, Sociedad Colombiana de Pedagogía, 1995. pág 27-34.

Bustamante, Guillermo. "Criterios contrastados sobre Proyecto Educativo InstitucionalPEl", en Pretextos Pedagógicos, No. 2, Bogotá, Sociedad Colombiana de Pedagogía, 1995.

"El PEI será lo que hagamos hoy por él”, en Educación y Cultura, No. 38. Bogotá, CEID-FECODE, agosto, 1995.

Cárdenas, Martha. "El PEI camino a la construcción de una escuela democrática", en Alegría de Enseñar No. 22, MEN-FES, Cali, 1995.

Castro, Luz Angela de y otros. "De los sueños a la realidad: la Escuela Nueva Delhi", en Alegría de Enseñar No. 22, MEN-FES Cali, 1995.

CEPAL-UNESCO. Educación y conocimiento: eje de la transformación productiva con equidad, Santiago, 1992.

Cerda, Ana María, Gabriel Aranquiz, Soledad Cid y Hugo Miranda. Los docentes y los procesos de descentralización pedagógica, Santiago, PIIE, 1994.

Consejería Presidencial para los Derechos Humanos. "Proyecto Educativo Institucional", en Democracia y Derechos Humanos, Bogotá, 1994.

Coy, María Elizabeth. "PEI Proyecto de transformación educativa", en Educación y Cultura, No. 38, Bogotá, CEID-FECODE, agosto. 1995.

Cubides, Hilda. "Una experiencia de construcción del PEI en el Colegio Carlos Arango Vélez", en Educación y Cultura, No. 38, p. 31.

DNP_-Presidencia de la República. "El Salto Educativo", en Revista Colombiana de Educación, No. 29, 1994.

Edwards, Verónica. Cómo aprende y cómo enseña el docente, PIIE. Instituto de Cooperación Iberoamericana. Santiago, 1992.

El currículum y la práctica pedagógica: análisis de dos contextos en la formación de docentes en Chile, Santiago, PIIE, 1995.

Escuela Filodehambre. "Aportes para la construcción del PEL", en Educación y Cultura, No. 38. p. 31.

Gajardo, Marcela (ed.). Cooperación internacional y desarrollo de la educación, Santiago, ASDI, ACCI, CLDE, 1994.

ICFES. Evaluación de matemáticas y lenguaje, Bogotá, 1994.

Instituto para el Desarrollo de la Democracia Luis Carlos Galán. Educación para la democracia en Colombia: carpeta de apoyos didácticos y mate-dales de lectura, Santafé de Bogotá, octubre de 1994.

Lucio, Ricardo. 'jQué tanto ayuda o entorpece el material disponible sobre el PEL?", en Educación y Cultura, No. 38, pág 19-24.

Martínez, Fanny. "Posible itinerario para trabajar por proyectos", en Alegría de Enseñar No. 22, MEN-FES, Cali, 1995. 
Ministerio de Educación Nacional. Reflexiones sobre los Proyectos Educativos Institucionales y guía para la construcción de planes operativos por parte de las comunidades educativas, Bogotá, 1994.

Lineamientos generales para la formación de los maestros, Bogotá. junio de 1995.

Moreno, María del Carmen. Innovaciones pedagógicas, Bogotá. Editorial Magisterio, 1994.

Niño, Libia Stella y otros. Evaluación, Proyecto Educativo y descentralización en la educación, Bogotá, Universidad Pedagógica Nacional.

Noguera, Carlos. "Algo más acerca de la Ley General de Educación", en Pretextos Pedagógicos, No. 2, Bogotá, Sociedad Colombiana de Pedagogía, 1995.

Peña, Luis Bernardo. "El Proyecto Educativo Institucional es una construcción social", en Alegría de Enseñar No. 22, págs 8 y 9.

Remedí, Eduardo. "Formas de interpelación en la construcción de una identidad: algunas propuestas de formación docente en las últimas décadas", en Cómo aprende y cómo enseña el docente, PILE, Santiago, 1992.

Rodríguez Rodríguez, José. El Proyecto Educativo: elementos para su diseño, Madrid, Alhambra Logman. 1990.

Salazar, Alba y otros. El Proyecto Educativo Institucional: una construcción de sentido: manual para el desarrollo del PEl, Bogotá. Editorial Santillana, 1995.

SABER. Informe sobre el Sistema Nacional de Evaluación, Bogotá, MEN, 1992.

Tezanos, Aracely de. "Mirar desde adentro", en Cómo aprende y cómo enseña el docente, PIIE, Santiago. 1992. 